\title{
Multi-Period Vendor Managed Inventory Systems
}

\author{
Rita Ribeiro* $\quad$ Helena R. Lourenço $^{\dagger}$
}

\begin{abstract}
In this paper we develop two models for an inventory system in which the distributor manages the inventory at the retailers location. These type of systems correspond to the Vendor Managed Inventory(VMI) systems described in the literature. These systems are very common in many different types of industries, such as retailing and manufacturing, although assuming different characteristics.

The objective of our model is to minimize total inventory cost for the distributor in a multi-period multi-retailer setting. The inventory system includes holding and stock-out costs and we study the case where an additional fixed setup cost is charged per delivery.

We construct a numerical experiment to analyze the model behavior and observe the impact of the characteristics of the model on the solutions.

Keywords: Vendor Managed Inventory Systems, Logistics.

JEL: C61, M19

\footnotetext{
*Department of Economics and Business and GREL-IET, Universitat Pompeu Fabra, Ramon Trias Fargas 25-27, 08005 Barcelona (Spain). Tel: 34-93 5422692, Fax: 34-93 5421746. E-mail: rita.ribeiro@upf.edu. This research has been partially financed by research grant from Ministério da Ciência e a Tecnologia, FCT, Prax is XXI/BD/21814/99.

${ }^{\dagger}$ Department of Economics and Business and GREL-IET, Universitat Pompeu Fabra, Ramon Trias Fargas 25-27, 08005 Barcelona (Spain). Tel: 34-93 5422753, Fax: 34-93 5421746. E-mail: helena.ramalhinho@upf.edu. This research has been partially financed by research grant MCYT BEC-2000 and GREL-IET.
} 


\section{Introduction}

Inventory systems vary throughout many dimensions, such as: the activities involved, parts studied, degrees of structural complexity, flows (information flows versus product flows) and the time horizon of the problem (one period, and finite and infinite horizons). See Zipkin (2000) for an overview of inventory systems

We can make a distinction between models with deterministic elements and stochastic elements. There are also some studies on how the introduction of inventory management technologies affects the inventory movements in some sectors, an example is the study by Worthington (1998).

The VMI system is a particular type of inventory management system that can be defined as a production/distribution and inventory control system where the manufacturer or the retailer makes the replenishment decisions for the consuming organization. This means the vendor monitors the buyers inventory levels (physically or via electronic messaging) and makes periodic resupply decisions regarding order quantities shipping and timing, Waller (1999).

Examples of industries where VMI policies are being used include: the petrochemical industry (gas stations), the grocery industry (supermarkets), the soft drink industry (vending machines) and the automotive industry (parts and components). There are several firm that have been adopting a type of a VMI system: Wal-Mart, Kmart, Dillard department stores, JCPenny are among the early adopters of VMI. But also in Hospital Materials Management, VMI achieved higher penetration, Dong (2002). Another example of a VMI system is the Campbell Soup continuous replenishment program, Cachon (1997).

Many advantages have been pointed out by several authors on the use of VMI systems: with VMI, greater coordination supports the supplier's need for smoother production without sacrificing the buyers service and stock objectives. Transportation costs are reduced, as well as truckload management, since the distributors do not respond automatically to orders as they are received, they can hold orders and decide when to execute them. Another attractive option pointed to VMI is a more efficient route planning.

Improving coordination of replenishment orders and deliveries across multiple customers helps to improve service by, for example, changing critical deliveries. Most of the inventory reduction achieved with VMI can be attributed to the more frequent inventory reviews, order intervals and deliveries. 
In our particular case, we are studying an inventory management system, with multiple and finite periods. The demand is stochastic, and the system has a single product and multiple-retailers. There is one warehouse or distributor that delivers a single product to multiple-retailers. A particular element is the week planning period, with no possibility of observing stock levels during that period, and at least one delivery during the planning period. The objective is to decide the delivery quantities and the delivery days for a set of vendor managed points such that the total inventory costs are minimized.

The organization of this paper is the following: In Section 2 a literature review on VMI will be briefly summarized. Then, on Section 3, we will present two Multi-Period Vendor Managed Inventory (MPVMI) models. The first model does not consider any setup cost and the second model includes this additional cost. In Section 4, we will perform a computational experiment on some examples. Finally, some conclusions and further research are drawn in Section 5.

\section{Literature review}

There exists a considerable amount of literature regarding inventory management problems and control systems. However, in this section we will focus our literature review on the work that has been done about VMI systems.

The VMI systems affect the Supply Chain (SC), Dong (2000) evaluates this effect and states that VMI always leads to a higher buyer's profit, but the supplier's profit varies. And, these benefits are obtainable only in a fully integrated SC.

Many references study the benefits of a VMI system over other inventory management systems. Waller (1999) studies the effect of the VMI in several environments. He states that, in this relationship, buyers relinquish control of key re-supply decisions and sometimes even transfer financial responsibility for the inventory to the supplier. The arrangement transfers the burden of asset management from the consuming organization to the vendor, who, may be obliged to meet specific customer service goal (usually some sort of in stock target). Some advantages of VMI are pointed, for example, reducing costs for each partner, reducing demand volatility, mitigating uncertainty of demand and solving the dilemma of conflicting performance measures. With VMI the frequency of replenishment is usually increased from monthly to weekly (or 
even daily), which benefits both sides. The vendor can make replenishment decisions and the buyer transfer inventory responsibilities.

Emigh (1999) presents an overview of VMI and its growing use throughout the retail industry. The author states that VMI is popular throughout the $\mathrm{SC}$ because it lets companies shift responsibility for inventory management to vendor in order to reduce overhead. Typically the manufacturer takes a daily review of inventory by pulling down EDI files from the distributor. The manufacturer then uses the inventory data to put together an anticipated order for the distributor. The apparel industry and hospital supplies, have been using non-automated VMI for decades, supermarkets have taken longer than department stores. VMI practitioners range from food manufacturers like Kraft Inc. to chain stores such as Wal-Mart stores to industries that use VMI automation like car and paper manufacturing industries.

Harrington (1996) describes the difference between VMI and consignment selling. Consignment selling allows manufacturers to place inventory at retailer's location, with the retailer never actually owning the product. Consignment selling is similar to VMI but differs in one key area. In traditional VMI, the retailer still owns the inventory; the manufacturer simply manages it. Consignment selling is the next step, the manufacturer owns the inventory and the retailer takes a percentage for providing shelf space and customers. In our work will refer our model as a VMI system although we will not make any distinction between who owns the inventory.

Andel (1996), suggests that many manufacturers are more adept to handling inventory than the retailers they supply. In a VMI relationship, vendors receive withdrawal and current balance information from the retailer and the vendor can arrange their shipments, build their loads, and cut their purchase orders to optimize their transportation and inventory requirements.

Dong (2002) evaluates how VMI affects a supply channel. VMI always leads to higher buyer's profit, but the supplier's profit varies. VMI is an effective SC strategy that can realize many of the benefits obtainable only in a fully integrated supply chain.

Aichlymayr (2000) shows some benefits of VMI and collaborative planning, forecasting and replenishment.

Other authors have studied the information sharing issue at VMI systems. Chueng (2002), considers a supply serving multiple retailers located in a close proximity, in his paper he examines the benefit of using customer demand information. Two types of SCs are considered that are often linked to VMI programs: the first uses information on the retailers' inventory posi- 
tion to coordinate shipments from the supplier to enjoy economies of scale; the second uses information for unloading the shipments to the retailers to rebalance their stocking positions.

Finally, some authors have worked on modeling VMI and optimizing replenishment policies. Disney (2002) defines VMI has a production/distribution and inventory control system where stock positions and demand rates are known across more than 1-echelon of the supply chain. VMI comes in many different forms described by terms such as Synchronized Consumer Response, Continuous Replenishment Programs, Efficient Consumer Response, and Rapid Replenishment, Collaborative Planning, Forecasting and Replenishment, Centralized Inventory Management. In his paper, the system is designed to minimize inventory-holding costs and adaptation related costs covering the need to ramp production up and down to meet perceived needs. In this work the system is designed for various different ratios of production adaptation costs and inventory holding costs and presents a decision support system that allows tuning the VMI system.

Fry (2001) models a type of VMI agreement where optimal replenishment and production policies for a supplier are found to be up-to-policies. The conclusion is that VMI performs better than a Retailer Managed Inventory (RMI) system (where the retailer managed inventory), in many settings but can perform worse in others, depending on the scenario and the contract parameters. For example, VMI performs better if outsourcing is very expensive or variance is high).

The VMI can also provide benefits by allowing the vendor to coordinate production and delivery, particularly in the case of multiple retailers, the following authors have studied this situation: Cheung and Lee (2002); Campbell (1999) studied a VMI policy where the objective was to minimize the average daily distribution cost during the planning period without causing stock-outs at any of the customers' location; Kleywest (2000), formulates an inventory routing problem for a VMI system where the supplier can measure the inventory level and decisions are made daily; Çetinkaya and Lee (2000) present an analytical model for coordinating inventory and transportation decisions in a VMI system where the vendor can hold the orders.

Our work can be included in this last group of literature. The basic idea is to develop a model for a VMI system with the characteristics of a week planning period, stochastic demand, unobservable stock levels and with a minimum of one visit per week.

The motivation of this paper is to respond to a strategic need and a 
growing tendency for planning deliveries and coordinating strategies within an integrated SC. Given a set of inventory managing costs we want to design the best delivery strategy taking into consideration the entire planning horizon. Even if, in practice, you may adapt your deliveries to customers and orders, you can obtain substantial gains by planning your inventory needs in advance. Another motivation for this work was based on the advantages that seem to exist when using a VMI system, in the coordination of the inventory management area with other management areas at the operational and strategic level, such as distribution, scheduling or location.

In the next section we will present the two models of VMI system.

\section{The MPVMI Models}

Our model consists of a VMI system with one supplier and multiple retailers. The distributor has the responsibility to decide when to visit the retailer's locations and how much to deliver. This particular model has the objective of designing the deliveries for a planning period, for a five day week period. One of the characteristics of the VMI systems is that the distributor is responsible for managing the inventory at the retailer point. He would like to minimize inventory handling costs while avoiding stock-out situations.

In a stock-out situation, a customer arrives at the selling point and there is no unit of product available at the site. This situation leads the distributor to incur a stock-out cost. These costs can be seen in two different ways: the cost of an emergency delivery to be able to serve the customer or an opportunity cost of a lost sale (in this case, assuming the customer leaves the retailers point and goes elsewhere). In both cases there is a strong cost, higher than the cost of handling an item on the shelves. So, one assumption of the model is that stock-out cost is always bigger than the holding cost and both are costs applicable per unit of product per day.

Next, we will list other assumptions of the model.

\subsection{Assumptions of the model}

- A week planning period is considered. We want to analyze a delivery strategy, we could have chosen two weeks or even one month but we have considered that one week is a common frequency in many industries and it is reasonable to plan for a week. 
- There are no handling stock costs at the warehouse, and we assume that there is enough amount of product (unlimited capacity). Keeping stock at the warehouse is always relatively cheep when comparing with the cost of keeping stock at retailers location, due to economies of scale and dispersion of locations. We will consider this cost as being part of production costs.

- Retailers have stochastic demand, but the distribution function of the demand for each customer and for each day is known.

- At the retailers location there are inventory handling costs and a cost for stock out, payed by the distributor.

- Each location is visited at least once a week. There is a periodic need for visiting the location for many possible reasons like: control reasons, checking stocks, shelves positions, promotion controls or other marketing activities. This assumption, in a way, reflects the cross-functional integration within a firm.

- The stock is only observed at the beginning of the planning period, and the decisions are made for the all planning horizon, independently of what occurs during the week. So, if demand exceeds the amount available there is a stock-out cost. There are no emergency deliveries or changes in the delivery plan due to the arrival of information. This does not mean that in practice these situations do not occur. But, we are analyzing a strategy for the best delivery policy with the information that we have, to minimize expected costs.

- The holding and stock-out costs only depend on quantities and not on retailers. They are both applied per unit of product per day and are the same for all retailers.

- We will consider two models: On the first model, there is no delivery cost, the only costs considered are the holding and stock-out cost at the retailers point. On the second model, there is an additional fixed setup cost per delivery made.

- The setup cost is a delivery cost per visit. This cost varies by retailers location and by day, but does not depend on the quantities delivered. 


\subsection{The MPVMI model with no setup cost}

In the first model, we consider that there is no cost associated with the delivery of the product to the retailer. The only costs considered are the costs of managing the inventory at the retailers point and the cost of a stockout situation. The distributor has to decide, for each day, how much to deliver and to which retailers, minimizing total costs. Delivering higher quantities increases the holding cost but decreases the possibilities of a stock-out.

\section{The costs of the problem are:}

- Inventory handling costs (holding costs) per unit of product at the retailers point, per day;

- Stock out costs at the retailers point, per unit lost, per day;

\section{The decisions to make are the following:}

- Decide, for each day, which points will be visited;

- How much to deliver at these points on each day.

\section{Objective function:}

Objective is to minimize the expected total cost at the end of the week; Min Weekly cost $=$ Inventory holding cost + Stock-out cost.

\section{Notation:}

$n=$ number of retailers, indexed from 1 to $n$;

$P=$ number of periods (in this case, 5 periods, from 0 to 4 );

$h=$ inventory carrying cost per unit;

$s=$ shortage cost per unit;

$\beta_{p i}=$ initial inventory at location $i$ on day $p, i=1, \ldots n ; p=1, \ldots, P$ and $\beta_{0 i}$ is known; 
$F_{i p}()=$. cumulative distribution function of the one period demand at retailer's location $i$, for day $p, i=1, \ldots n$ and $p=1, \ldots, P$;

$f_{i p}=$ distribution function of the one period demand at retailer's location $i$, for day $p, i=1, \ldots n$ and $p=1, \ldots, P$;

$t_{i p}=$ random variable representing the demand of retailer $i$ on day $p$, $i=1, \ldots n$ and $p=1, \ldots, P$;

\section{Decision Variables:}

$$
\begin{aligned}
& w_{i}^{p}=\text { amount delivered to retailer } i \text { on day } p \\
& y_{i p}=\left\{\begin{array}{lll}
1 & \text { if } & \text { retailer } i \text { is visited on day } p \\
0 & \text { if } & \text { otherwise }
\end{array}\right.
\end{aligned}
$$

\section{Inventory cost:}

$$
\sum_{i \in B} \sum_{p}\left[\begin{array}{c}
h \sum_{t_{i p}=\mathbf{0}}^{\beta_{i p}+w_{i p}}\left(\beta_{i p}+w_{i p}-t_{i p}\right) f_{i p}\left(t_{i p}\right) d t_{i p} \\
+s \sum_{\beta_{i p}+w_{i p}}^{\infty}\left(t_{i p}-\beta_{i p}-w_{i p}\right) f_{i p}\left(t_{i p}\right) d t_{i p}
\end{array}\right]
$$

For each day and for each retailer, the inventory cost is equal to the the sum of the expected inventory holding and stock-out costs.

For each period, if demand exceeds the initial stock of that period plus the quantity delivered, there is a cost $s$ per unit exceeded. Otherwise, if the demand is smaller than the quantity delivered and the initial stock, the vendor incurs in a holding cost $h$, per unit of end stock.

This expression applies for the case where demand is a discrete variable. Since the representation of the probability distribution is difficult to find, particularly when demand ranges over a large number of possible values, the discrete random variable is often approximated by a continuous random variable (Hillier and Lieberman (1995)). Furthermore, when demand ranges from over a large number of possible values, this approximation will generally yield a nearly exact value of the optimal amount. In addition, when discrete demand is used, the resulting expression may become slightly more difficult 
to solve analytically. So, we will approximate the demand as a continuous random variable and at the end we approximate the solution to the closest integer.

Let $I_{i p}\left(w_{i p}\right)$ represent the inventory managing cost of location $i$ on day $p$.

$$
\begin{aligned}
& \sum_{i} \sum_{p} I_{i p}\left(w_{i p}\right) \\
= & \sum_{i} \sum_{p}\left[\begin{array}{c}
h \int_{0}^{\beta_{i p}+w_{i p}}\left(\beta_{i p}+w_{i p}-t_{i p}\right) f_{i p}\left(t_{i p}\right) d t_{i p} \\
+s \int_{\beta_{i p}+w_{i p}}^{\infty}\left(t_{i p}-\beta_{i p}-w_{i p}\right) f_{i p}\left(t_{i p}\right) d t_{i p}
\end{array}\right]
\end{aligned}
$$

The problem can be stated as follows:

$$
\operatorname{Min} \sum_{i} \sum_{p} I_{i p}\left(w_{i p}\right)
$$

Subject to:

$$
\begin{aligned}
& w_{i p} \leq M \times y_{i p}, \quad \forall i, p \\
& 1 \leq \sum_{p} y_{i p}, \quad \forall i \\
& w_{i p} \geq 0, \quad \forall i, p \\
& y_{i p} \in\{0,1\} \quad \forall i, p
\end{aligned}
$$

The meaning of the above constraints is:

(3) If the quantity to be delivered is positive, than that location has to be visited on that day.

(4) The VMI retailers are visited at least once a week.

(5) The variable $w_{i p}$, that represents the quantity delivered to a retailer on a given day is always greater or equal to 0 .

(6) This constraint defines $y_{i k}^{p}$ as binary.

At this point, we have an additional problem with the initial inventory: The initial stock in only observed at the beginning of the planning horizon. Then, we have to decide the delivery days and quantities without considering 
any information of the stocks during the period. We observe the initial inventory of each retailer at the beginning of period 0 , so the initial stock of the future periods is also a random variable, since it depends on demand of all the previous periods.

At each period, if there is a stock-out, a cost is incurred and the initial stock of the following period will be zero. There is an interdependence between all periods, the initial inventory for each period is:

$$
\begin{aligned}
& \beta_{i 0}=u_{i}>0 \text {, observed } \\
& \beta_{i 1}=\operatorname{Max}\left\{0, u_{i}+w_{i 0}-t_{i 0}\right\} \\
& \beta_{i 2}=\operatorname{Max}\left\{0, \beta_{i 1}+w_{i 1}-t_{i 1}\right\}=\operatorname{Max}\left\{0, \operatorname{Max}\left\{0, u_{i}+w_{i \mathbf{0}}-t_{i \mathbf{0}}\right\}+w_{i 1}-t_{i 1}\right\} \\
& \beta_{i 3}=\operatorname{Max}\left\{0, \beta_{i 2}+w_{i 2}-t_{i 2}\right\}= \\
& =\operatorname{Max}\left\{0, \operatorname{Max}\left\{\begin{array}{c}
0, \operatorname{Max}\left\{0, u_{i}+w_{i \mathbf{0}}-t_{i \mathbf{0}}\right\}+ \\
w_{i 1}-t_{i 1}
\end{array}\right\}+w_{i 2}-t_{i 2}\right\} \\
& \beta_{i 4}=\operatorname{Max}\left\{0, \beta_{i 3}+w_{i 3}-t_{i 3}\right\}=
\end{aligned}
$$

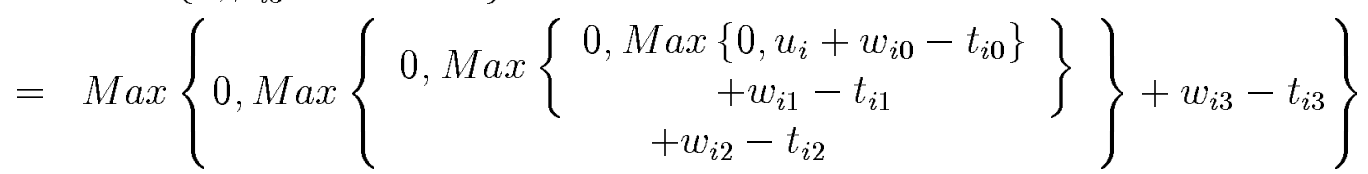

So, the initial stock of period $p$ will be a function of the initial stock at period 0 and of the quantities delivered and demand of all previous periods.

$$
\beta_{i p}\left(u_{i}, w_{i \mathbf{0}}, \ldots, w_{i p-1}, t_{i \mathbf{0}}, \ldots t_{i p-1}\right)
$$

In this model we will assumed a stochastic demand that follows an exponential distribution known by the distributor, with the form:

$$
f\left(t_{i p}\right)=a_{i p} e^{-a_{i p} t_{i p}}
$$

with the average demand $1 / a_{i p}$. There are many different statistical distribtuions to choose from when working with inventory control. The exponential distribution function is commonly used to represent the demand variation in inventory models. See Lidke and Malstrom (1987) and Snyder (1984).

It makes sense that in some cases demand varies from day to day and from retailer to retailer, we have considered a demand function parameter for each retailer and for each day. 
We need to calculate the inventory cost for each period. So, for each period we need to consider all the possible scenarios of the previous periods initial inventory. This is, for period 0 , we know the initial inventory, for period 1 we need to consider two possibilities: stock-out at the end of period zero (zero initial inventory at period 1) and positive stock at the end of period 0 (initial stock at period 1 positive). Extending this for all the periods we obtain the complete inventory cost function.

$$
h \int_{0}^{\beta_{i p}+w_{i p}}\left(\beta_{i p}+w_{i p}-t_{i p}\right) f_{i p}\left(t_{i p}\right) d t_{i p}+s \int_{\beta_{i p}+w_{i p}}^{\infty}\left(t_{i p}-\beta_{i p}-w_{i p}\right) f_{i p}\left(t_{i p}\right) d t_{i p}
$$

We will use the Heaviside function to simplify the expression,

substituing:

$$
1\left(t_{i p}<\beta_{i p}+w_{i p}\right)=\left\{\begin{array}{ccc}
1 & \text { if } & t_{i p}-\beta_{i p}-w_{i p}<0 \\
0 & \text { if } & \text { otherwise }
\end{array}\right.
$$

becomes:

$$
\int_{0}^{\infty}\left[\begin{array}{c}
h \times 1\left(t_{i p}<\beta_{i p}+w_{i p}\right)\left(\beta_{i p}+w_{i p}-t_{i p}\right) \\
+s \times 1\left(t_{i p}>\beta_{i p}+w_{i p}\right)\left(t_{i p}-\beta_{i p}-w_{i p}\right)
\end{array}\right] f\left(t_{i p}\right) d t_{i p}
$$

For simplicity, in the next sections we will derive the expressions for one retailer only, then add for all retailers.

Since obtaining the expression for the inventory cost function is complex we will first obtain the expressions for the inventory cost expression for each period separately, the complete cost function is the sum of the inventory cost of each day.

For the first period, the inventory cost, $I_{0}$, is:

\section{Period 0:}

$$
\begin{aligned}
I_{0} & =\int_{0}^{\infty}\left[\begin{array}{c}
h * 1\left(u+w_{0}-t_{0}\right) *\left(u+w_{0}-t_{0}\right) \\
-s * 1\left(-u-w_{0}+t_{0}\right) *\left(u+w_{0}-t_{0}\right)
\end{array}\right] f\left(t_{0}\right) d t_{0} \\
& =\exp \left(-a_{0} *\left(u+w_{0}\right)\right) *(s+h) / a_{0}+h *\left(a_{0} * u+a_{0} * w_{0}-1\right) / a_{0}
\end{aligned}
$$

$I_{0}$ is the inventory cost in period 0 . It is equal to a holding cost of $h *\left(u+w_{0}-t_{0}\right)$ if $u+w_{0}>t_{0}$ and a shortage cost of $s *\left(t_{0}-u-w_{0}\right)$ if $u+w_{0}<t_{0}$. 
For period 1 we will have to consider the 2 possible outcomes of period 0 .

\section{Period 1:}

$$
\begin{gathered}
I_{1}=\int_{0}^{\infty} \int_{0}^{\infty}\left[\begin{array}{c}
1\left(u+w_{0}-t_{0}\right) *\left(h * 1\left(u+w_{0}+w_{1}-t_{0}-t_{1}\right)\right. \\
*\left(u+w_{0}+w_{1}-t_{0}-t_{1}\right) \\
-s * 1\left(-u-w_{0}-w_{1}+t_{0}+t_{1}\right) \\
\left.*\left(u+w_{0}+w_{1}-t_{0}-t_{1}\right)\right) \\
+1\left(-u-w_{0}+t_{0}\right) *\left(h * 1\left(w_{1}-t_{1}\right) *\left(w_{1}-t_{1}\right)\right. \\
\left.-s * 1\left(-w_{1}+t_{1}\right) *\left(w_{1}-t_{1}\right)\right)
\end{array}\right] f\left(t_{0}\right) f\left(t_{1}\right) d t_{0} d t_{1} \\
I_{1}= \\
\quad\left(\left(\alpha_{1} * u+\alpha_{1} * w_{0}+1\right) / \alpha_{1} * h+\left(\alpha_{1} * u+\alpha_{1} * w_{0}\right.\right. \\
\left.+1) / \alpha_{1} * s\right) * \exp \left(-\alpha_{1} *\left(u+w_{0}+w_{1}\right)\right) \\
+1 / \alpha_{1} * h * \exp \left(-\alpha_{1} *\left(u+w_{0}\right)\right) \\
+\left(-2+\alpha_{1} * w_{1}+\alpha_{1} * u+\alpha_{1} * w_{0}\right) / \alpha_{1} * h
\end{gathered}
$$

$I_{1}$ is the inventory cost of period 1 . It is the inventory cost considering two possibilities:

- Positive stock in period 0:

$$
\begin{aligned}
& 1\left(u+w_{0}-t_{0}\right) *\left(h * 1\left(u+w_{0}+w_{1}-t_{0}-t_{1}\right) *\left(u+w_{0}+w_{1}-t_{0}-t_{1}\right)-\right. \\
& \left.s * 1\left(-u-w_{0}-w_{1}+t_{0}+t_{1}\right) *\left(u+w_{0}+w_{1}-t_{0}-t_{1}\right)\right)
\end{aligned}
$$

- Stock-out in period 0:

$$
1\left(-u-w_{0}+t_{0}\right) *\left(h * 1\left(w_{1}-t_{1}\right) *\left(w_{1}-t_{1}\right)-s * 1\left(-w_{1}+t_{1}\right) *\left(w_{1}-t_{1}\right)\right)
$$

For the third period we would have to consider 4 possibilities, for the fourth period 8 and 16 for the fifth.

The analytical expression for the inventory cost function of all the periods is in Appendix 1.

The total inventory cost will be the sum of the cost of each period:

$$
\sum_{p=0}^{4} I_{p}
$$


The inventory cost function is a non-linear function. The first order derivatives of the inventory cost function are also non-linear functions. And, we also have the constraint that the w's can not assume negative values. So, to minimize the function we will use the Gauss-Newton's approximation methods (Bertsekas (1995)).

The result obtained after solving this model is a delivery policy which implies that the distributor visits a location almost every day. In some cases, if initial stock is sufficiently high, then the best solution will be to deliver after this stock has ran out. Nonetheless, it makes sense to include a cost per delivery that might change the above delivery policy. This cost, in a way, reflects the transportation cost. This is what we will consider in the next model.

\subsection{The model with setup cost}

Consider now that there is an additional cost, a setup cost per delivery. This setup cost is a fixed charge, independent of the quantity delivered, that will vary from retailer to retailer and eventually from day to day and is applied whenever a deliver is made to a retailer. It does not depend on the quantities delivered, it corresponds to the cost of preparing the order, transportation cost and delivery cost.

The model will again minimize total cost, but this time it includes holding and stock-out costs per unit of product per day and the setup cost per day and per retailer visited.

The setup cost varies from retailer and day, this assumption is justified when looking at the setup cost as a transportation cost. Customer far away from the warehouse or with more difficult access (ex. downtown areas) have associated a higher delivery cost.

Delivery costs might also vary by day, looking again in terms of transportation and analyzing the group of retailers to be served, a retailer away from the warehouse but closer to other group of retailer being visited has a lower cost than if he is isolated. Another aspect is the number of routes, a retailer served on a given day might be included in a tour which is costly than if it is included on another day.

Let us now introduce the setup cost in the inventory function:

$z_{i p}=$ setup cost of delivering to retailer $i$ on day $p$, with $i=1, \ldots n$ and $p=1, \ldots, P$ 
The inventory cost function with setup cost $(I S)$ is:

$$
\begin{aligned}
I S & = \\
& =\sum_{i} \sum_{p}\left[\begin{array}{c}
1\left(w_{i p}<0\right) \times z_{i p} \\
+h \int_{0}^{\beta_{i p}+w_{i p}}\left(\beta_{i p}+w_{i p}-t_{i p}\right) f_{i p}\left(t_{i p}\right) d t_{i p} \\
+s \int_{\beta_{i p}+w_{i p}}^{\infty}\left(t_{i p}-\beta_{i p}-w_{i p}\right) f_{i p}\left(t_{i p}\right) d t_{i p}
\end{array}\right]
\end{aligned}
$$

with

$$
1\left(w_{i p}>0\right)=\left\{\begin{array}{ccc}
1 & \text { if } & w_{i p}>0 \\
0 & \text { if } & \text { otherwise }
\end{array}\right.
$$

Let us start by analyzing the inventory cost for only one retailer $i$ and one period $p$ :

$$
\begin{array}{ccc}
z_{i p}+I\left(w_{i p}^{*}\right) & \text { if } & w_{i p}^{*}>0 \\
I\left(w_{i p}^{*}\right) & \text { if } & w_{i p}^{*}=0
\end{array}
$$

To solve this problem:

First, let $w_{i p}^{*}$ be the delivering quantity that Minimizes $I_{i p}$

If we deliver, it becomes: $I\left(w_{i p}^{*}\right)+z_{i p}$

So we have to compare $I\left(w_{i p}=0\right)$ with $I\left(w_{i p}^{*}\right)+z_{i p}$

Let $r_{i p}^{*}$ be the value that equals $I\left(w_{i p}=0\right)=I\left(w_{i p}^{*}\right)+z_{i p}$

Then the optimal cost will be:

$$
\begin{array}{lll}
z+I\left(w_{i p}^{*}\right) & \text { if } & \beta_{i p} \leq r_{i p}^{*} \\
I\left(w_{i p}=0\right) & \text { if } & \beta_{i p}>r_{i p}^{*}
\end{array}
$$

If we try to extend this problem to more than one period the decision tree will increase, see Figure (1) for the decision tree of all the planning horizon. 


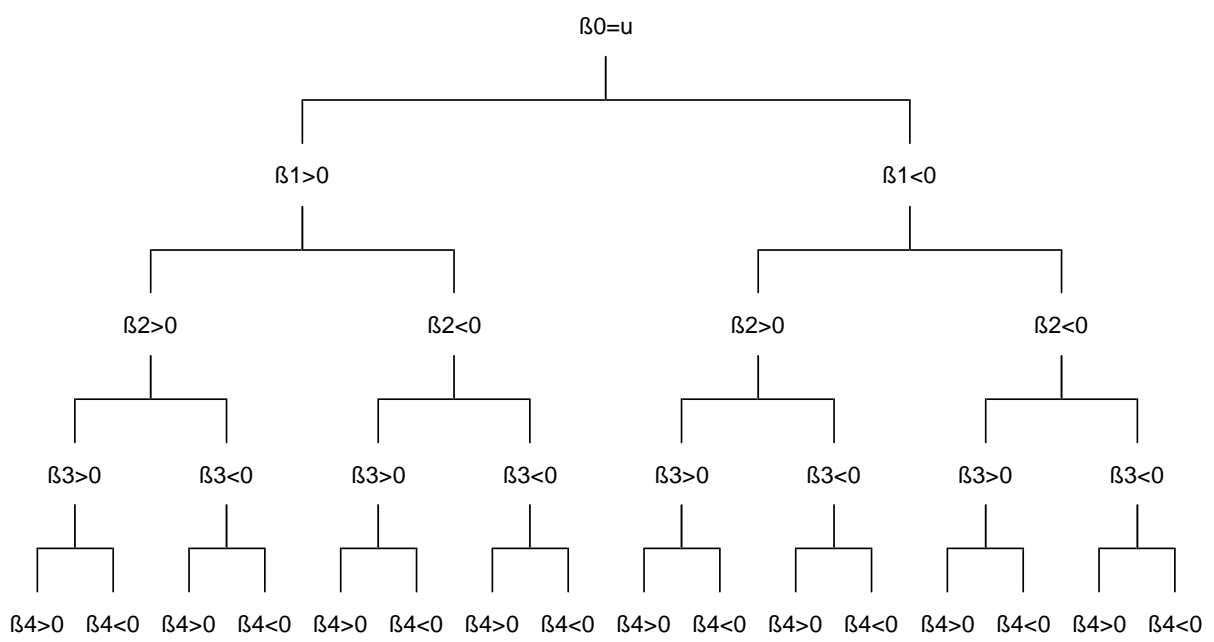

Figure 1: Decision tree for the MPVMI model with setup cost.

To solve this problem becomes a complex task. The solution we propose is to analyze the best delivery quantities for all the possible combinations of delivery days and choose the solution with minimum total costs (inventory + stock-out + setup costs).

So, for each retailer given the setup cost for each day of the week, we calculate the best delivery quantities for all the possible combinations of days.

We do not consider the case when there is no delivery since one of the assumptions is that at least once a week the retailer has to be visited.

The procedure to solve the problem is the following :

- Minimize the inventory cost for each combination of delivery days.

- Obtain the best delivery quantities for that combination.

- Calculate the total inventory cost by adding to the inventory cost the corresponding setup-cost.

- Choose the combination and quantities that gives the best solution (minimum total inventory cost).

- Repeat the procedure for each retailer.

Another point is the constraint that imposes only positive values for the deliveries, which transforms the problem into a constrained minimization problem. 
The solution to the first model, with no setup cost corresponds to the combination where we considered that is possible to delivery every day of the planning period ( 5 days). The second model, with the setup costs, chooses the best solution from all the possible combinations, including delivering every day.

In the next section, we will describe the computational experiment that we have done on several examples, and show the main results.

\section{Computational experiment}

In this section, we will present a numerical study on the two MPVMI models with and without setup costs. We will refer to Model 1 (M1) for the MPVMI with no setup cost and Model 2 (M2) to the MPVMI with setup cost.

The objective of this experiment is to compare the solutions of the two models in terms of costs and delivery days.

Results show that optimal solutions depend on the type of demand and on the setup cost. When introducing a setup cost, the average number of delivery days decreases while total costs increase.

Next, we will explain the data used and analyze some important results of this experiment.

\subsection{The data}

For the computational experiment we have generated several instances that can be divided in different groups according to their size, measured by the number of retailers $(10,50,100,200)$, type of setup cost (equal every day versus different for each day, and low versus high) and type of demand (same every day and different every day).

For the demand data we have generated the distribution function parameter $\alpha_{i p}$. So, for each retailer $i$ and day $p$ we have generated a value for $\alpha_{i p}$. These values were randomly chosen from a Normal distribution with mean 50 and standard deviation 20 between retailers. For instances with demand varying from day to day, the mean was the same as before, but the standard deviation between days was equal to 2 .

The initial stock for each retailer was generated by a random uniform distribution between 0 and 50. Finally, the setup cost was generated for each retailer and for each day of the week, following a uniform distribution 
between 10 and 200 for high setup cost and between 5 and 50 for low setup cost. The total number of cases analyzed was 160 .

\subsection{Analysis of the results}

We can start by analyzing and comparing the Inventory Cost. In Table 1, we have the Inventory Cost for M1 and M2, with and without the setup cost ${ }^{1}$, separated by size and type of setup cost. The cost in this table is an average of all instances with the same size.

\begin{tabular}{llllll}
\hline $\boldsymbol{N}$ & Setup & M1 & M1+setup & M2 & M2+setup \\
\hline \hline 10 & HS & 6211,60 & 11039,55 & 7193,79 & 8270,99 \\
& LS & 6211,60 & 7335,95 & 6318,67 & 6989,07 \\
\hline 50 & HS & 30304,26 & 55249,71 & 35309,20 & 40974,90 \\
& LS & 30304,26 & 36798,16 & 31431,37 & 34983,87 \\
\hline 100 & HS & 60588,02 & 110789,07 & 70499,63 & 81778,17 \\
& LS & 60588,02 & 73643,97 & 62803,86 & 69832,36 \\
\hline 200 & HS & 120429,09 & 222111,27 & 140718,38 & 163254,89 \\
& LS & 120429,09 & 146841,40 & 125169,50 & 139334,50 \\
\hline
\end{tabular}

Table 1: Average Inventory Costs for M1 and M2 for Low (LS) and High (HS) setup costs.

From Table 1 we can observe that M2 has always an Inventory Cost (free of the setup cost) greater than M1. However, M1 and M2 are closer to each other when the setup cost is low. These results were expected since if we are using an additional cost in the decision process the solution will be worst. The opposite happens when looking at both models when adding the correspondent setup cost, M1 will have always the highest total cost.

\footnotetext{
${ }^{1}$ Although we have assumed that in M1 there are no setup costs, we have added to M1 the setup cost that would be applied if we were delivering on the days proposed by M1. This is done so that we can compare both models.
} 
In Table 2, the instances were separated into two groups: the group with a setup cost that is equal every day and a group with setup cost that differs each day.

\begin{tabular}{|c|c|c|c|c|c|}
\hline$N$ & Low Setup & M1 & M1+setup & M2 & M2+setup \\
\hline \multirow[t]{2}{*}{10} & $=$ & 6211,60 & 7314,10 & 6298,71 & 6997,01 \\
\hline & $\neq$ & 6211,60 & 7357,80 & 6338,63 & 6981,13 \\
\hline \multirow[t]{2}{*}{50} & $=$ & 30304,26 & 36592,86 & 31176,13 & 34944,93 \\
\hline & $\neq$ & 30304,26 & 37003,46 & 31686,60 & 35022,80 \\
\hline \multirow[t]{2}{*}{100} & $=$ & 60588,02 & 73531,22 & 62465,64 & 70004,54 \\
\hline & $\neq$ & 60588,02 & 73756,72 & 63142,08 & 69660,18 \\
\hline \multirow[t]{2}{*}{200} & $=$ & 120429,09 & 146875,40 & 124546,33 & 139814,12 \\
\hline & $\neq$ & 120429,09 & 146807,40 & 125792,68 & 138854,89 \\
\hline$N$ & High Setup & M1 & M1+setup & M2 & M2+setup \\
\hline \multirow[t]{2}{*}{10} & $=$ & 6211,60 & 10994,00 & 7142,08 & 8441,98 \\
\hline & $\neq$ & 6211,60 & 11085,10 & 7245,50 & 8100,00 \\
\hline \multirow[t]{2}{*}{50} & $=$ & 30304,26 & 54572,06 & 34918,82 & 41780,72 \\
\hline & $\neq$ & 30304,26 & 55927,36 & 35699,58 & 40169,08 \\
\hline \multirow[t]{2}{*}{100} & $=$ & 60588,02 & 110704,02 & 70171,28 & 83733,48 \\
\hline & $\neq$ & 60588,02 & 110874,12 & 70827,97 & 79822,86 \\
\hline \multirow[t]{2}{*}{200} & $=$ & 120429,09 & 223041,82 & 140034,83 & 167567,55 \\
\hline & $\neq$ & 120429,09 & 221180,72 & 141401,92 & 158942,23 \\
\hline
\end{tabular}

Table 2: Average inventory cost for M1 and M2 with equal setup cost (=) every day versus different setup cost $(\neq)$ for each day.

The results show that, when setup cost differs from day to day, then the best solution of M2 has a higher cost than when the setup cost is equal 
every day. This can be explained by the higher influence setup cost plays on solutions, if it varies, solutions adjust more to these differences.

We have also analyzed and separated the instances by the type of demand. In Table 3, we have the inventory cost for M1 and M2 with equal demand every day and different demand for each day. The same relation applies for the difference between M1 and M2. This difference is smaller when demand varies from day to day. This is, the model leads to lower costs by playing with the variations on the expected demand.

\begin{tabular}{cccccc}
\hline $\boldsymbol{N}$ & Demand & M1 & M1+setup & M2 & M2+setup \\
\hline \hline 10 & $=$ & 6494,42 & 9512,12 & 7128,76 & 8060,41 \\
& $\neq$ & 5928,78 & 8863,38 & 6383,69 & 7199,64 \\
\hline 50 & $=$ & 29787,64 & 45563,24 & 33150,73 & 37696,18 \\
& $\neq$ & 30820,88 & 46484,63 & 33589,84 & 38262,59 \\
\hline \multirow{2}{*}{100} & $=$ & 60830,22 & 92653,16 & 67667,79 & 76941,63 \\
& $\neq$ & 60345,83 & 91779,88 & 65635,70 & 74668,90 \\
\hline \multirow{2}{*}{200} & $=$ & 121550,58 & 185917,46 & 135340,07 & 153995,22 \\
& $\neq$ & 119307,60 & 183035,21 & 130547,82 & 148594,18 \\
\hline
\end{tabular}

Table 3: Average Inventory Cost for M1 and M2 with equal demand (=) every day versus different demand $(\neq)$ for each day.

In Table 4, we can see the average number of delivery days in the solutions of M1 and M2. The first observation is that the number of delivery days does not depend on the size of the problem (retailer have an inventory cost that is independent of other retailers). As expected M1 has high delivery days in the planning period, on average 4,7 days per week (in 5 days). When introducing a setup cost in the decision process (M2), the number of days a retailer is visited during the planning horizon falls to an average of 2,3 days a week. 


\begin{tabular}{lcc}
\hline $\boldsymbol{N}$ & $\boldsymbol{M 1}$ & M2 \\
\hline \hline 10 & 4,70 & 2,41 \\
\hline 50 & 4,76 & 2,37 \\
\hline 100 & 4,63 & 2,23 \\
\hline 200 & 4,78 & 2,32 \\
\hline
\end{tabular}

Table 4: Average number of delivery days per week in M1 and M2.

And, if we analyze the two instances separated by high and low setup cost, in M2, the average number of delivery days goes from an average of 3 days per week for the Low setup cost case to an average of 1,6 delivery days for the case of high setup costs. There is a strong relation between the magnitude of the setup cost and the number of delivery days per week in the solution.

\begin{tabular}{llll}
\hline $\boldsymbol{N}$ & Setup & M1 & M2 \\
\hline \hline 10 & HS & 4,70 & 1,58 \\
& LS & 4,70 & 3,25 \\
\hline 50 & HS & 4,76 & 1,64 \\
& LS & 4,76 & 3,10 \\
\hline 100 & HS & 4,64 & 1,74 \\
& LS & 4,62 & 2,72 \\
\hline 200 & HS & 4,78 & 1,61 \\
& LS & 4,78 & 3,03 \\
\hline
\end{tabular}

Table 5: Average number of delivery days in M1 and M2 for the cases of Low (LS) and High (HS) setup cost. 
The relation between the two models can also be observed in Figure (2). In this graph we have the inventory cost for M1 and M2 with and without setup cost, for a set of 20 retailers with equal demand and low and equal setup cost. As can be seen, the inventory cost of M1 is always the lowest if free of setup costs and the highest costs if we add the correspondent setup cost.

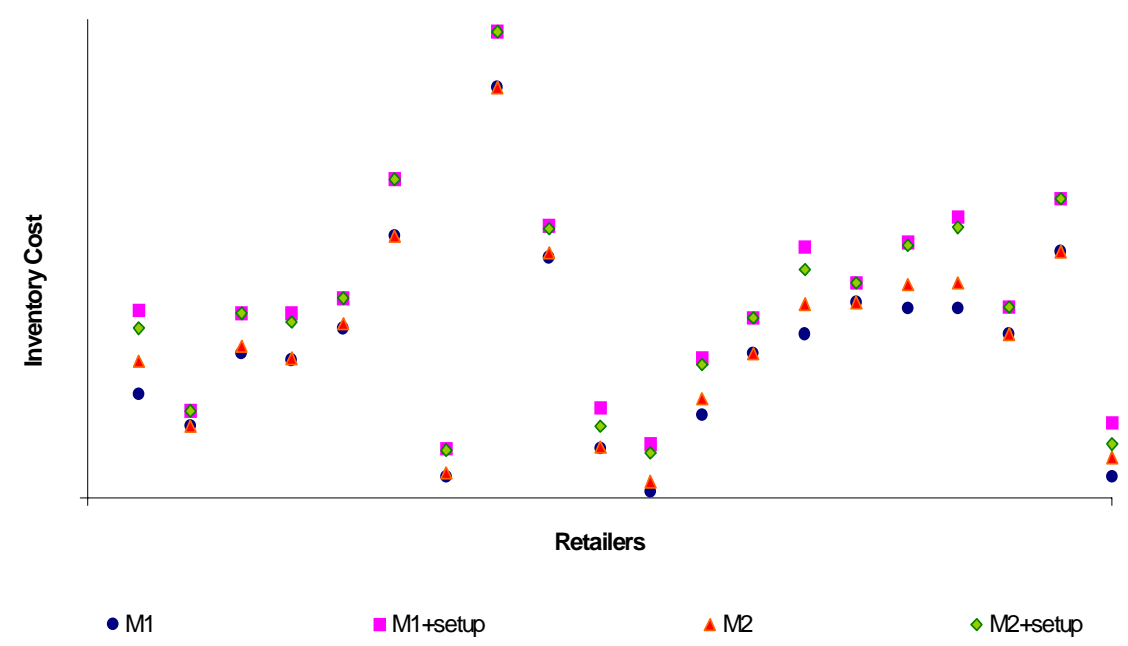

Figure 2: Inventory Costs for M1 and M2 for a group of 20 Retailers.

Finally, concerning the run time of the algorithm, in Table 6, we see that it is proportional to the size of the problem (number of retailers), this result is due to independence of the inventory cost function among retailers. The run time, in these particular models, does not have major implications since it takes an average of 0,38 seconds per retailer to solve a week planning problem. 


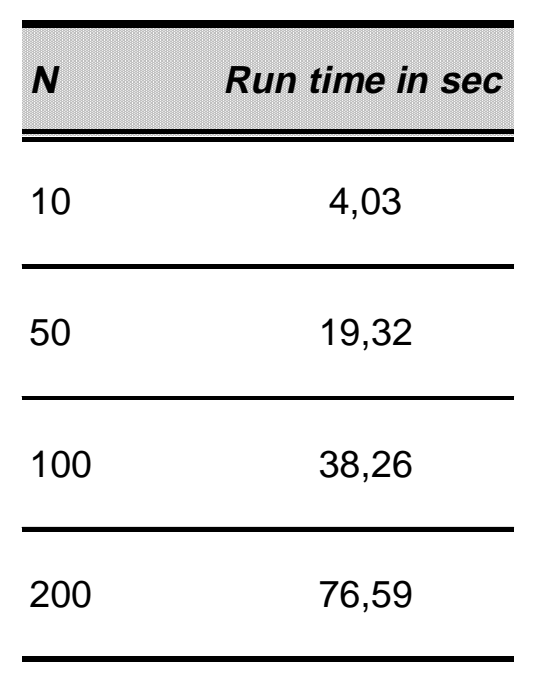

Table 6: Run time in seconds for M1+M2.

The objective of the above numerical experiment is twofold: In the first place analyze the two models in terms of inventory cost and number of delivery days. Secondly, to give an insight on the effect the delivery frequency can have in terms of costs. There is a clear trade-off between delivery days and inventory costs and the decision maker might evaluate what is the effect, for example, in terms of marketing or other control policies, of not visiting a retailer on a given day.

\section{Conclusions}

Inside the trend to a wider cooperation and integration of logistics processes is an initiative called the VMI system. In a VMI system the supplier makes the inventory decisions for the consuming organization.

The VMI system has gained importance during the last few years. The introduction of technologies for information sharing and coordination has contributed for the success of those policies in many industries. Nonetheless, there are still gaps to fill in the research in the area of modeling these agreements and using algorithms to strategically plan the inventory managing systems.

The motivation of this work arrives from the need to strategically respond to the growing tendency for planning deliveries and coordinating strategies within an integrated SC. And also, from the advantages that seem to exist 
when using a VMI system, in the coordination of the inventory management area with other management areas at the operational and strategic level, such as distribution, scheduling or location.

This work presents two inventory models: the MPVMI with no setup cost and the MPVMI with setup costs. In these models it is the responsibility of the distributor to manage the inventory at the retailers' location. The distributor does not know the demand of the product and only observes the initial stock at the beginning of the planning period. He has to decide how much and when to deliver to each of its retailers while trying to minimize total inventory costs. We have done a computational experiment and the results show that, by introducing a setup cost, the number of deliveries per planning period are reduced while holding plus stock-out costs increase. When comparing the inventory costs including setup cost, the inventory model solved without considering the setup costs (M1), has higher total costs. The impact of his trade-off depends on the type of demand and level of setup cost used.

Our models gain interest in the above setting if we consider them as tools for the coordination with other areas in the SC. One example is to consider the setup cost as a transportation cost and to use this model to manage and define policies for a large scope of the supply chain management, in this particular case the transportation and inventory management. This is commonly known as the inventory-routing model and will be the subject of our future research: design a model for the integration of this VMI system with other transportation issues, such as the routing of vehicles for the distribution the products to retailers.

\section{References}

[1] Aichlymayr, M. (2000). "DC mart: Who manages inventory in a value chain?" Tranportation \& Distribution 41(10): 60-66.

[2] Andel, T. (1996). "Manage Inventory, Own Information." Tranportation \& Distribution: 54-58.

[3] Bertsekas, D. P. (1995). Non-Linear Programming. Belmont Massachusettes, Athena Scientific. 
[4] Cachon, G.P. and Fisher, M. (1997). "Campbell Soup's continuous replenishment program: evaluation and enhanced inventory decision rules." Production and Operations Management 6(3): 266-276.

[5] Campbell, A., Clarke, L. and Savelsbergh (1999). "An inventory routing problem." working paper.

[6] Cheung, K.L. and Lee, H.L. (2002). "The inventory Benefit of shipment Coordination and stock rebalancing in a supply chain." Management Science 48(2): 300-306.

[7] Disney, S.M. and Towill, D.T. (2002). "A procedure for the optimization of the dynamic response of a Vendor Managed Inventory system." Computers \& Industrial Engineering 43: 27-58.

[8] Dong, Y. and Xu, K. (2002). "A supply chain model of vendor managed inventory." tranportation Research. Part E 38: 75-95.

[9] Emigh, J. (1999). "Vendor Managed Inventory." Computers World: 52.

[10] Fry, M.J., Kapuscinski, R. and Olsen, T.L. (2001). "Coodinating Production and Delivery Under a $(z, Z)$-Type Vendor-Managed Inventory Contract." Manufacturing \& Service Operations Management 3(2): 151173.

[11] Harrington, L. (1996). "Consignment selling: Trend or another World?" Tranportation \& Distribution 37(6): 45.

[12] Hillier, F.S. and Lieberman, G.J. (1995). Introduction to Operations Research, McGraw-Hill International Series.

[13] Lidke, S. L. and E. M. Malstrom (1987). "A recursive computer algorithm fo determining joint probability inventory distributions." Computers \& Industrial Engineering 12(2): 105-116.

[14] Kleywegt, A.J., Nori, V.S. and Savelsbergh, M.W.P. (1998). "A computational approach for the inventory routing problem." working paper.

[15] Snyder, R. D. (1984). "Inventory control with gamma probability distribution." European Journal of Operational Research 17(3): 373-381. 
[16] Waller, M., Johnson, M.E. and Davis, T. (1999). "Vendor-managed inventory in the retail supply chain." Journal of Business Logistics 20(1): 183-203.

[17] Worthington, P.R. (1998). "The increasing importance of retailers' inventories." Economic Prespectives 22(3): 2-13.

[18] Zipkin, P.H. (2000). Foundations of Inventory Management, McGrawHill International Editions. 


\section{Appendix 1: Inventory cost function for each period, for one retailer.}

Period 0:

$$
I_{0}=\exp \left(-a_{0} *\left(u+w_{0}\right)\right) *(s+h) / a_{0}+h *\left(a_{0} * u+a_{0} * w_{0}-1\right) / a_{0}
$$

Period 1:

$$
\begin{aligned}
I_{1}= & \left(\left(\alpha_{1} * u+\alpha_{1} * w_{0}+1\right) / \alpha_{1} * h+\left(\alpha_{1} * u+\alpha_{1} * w_{0}\right.\right. \\
& \left.+1) / \alpha_{1} * s\right) * \exp \left(-\alpha_{1} *\left(u+w_{0}+w_{1}\right)\right) \\
& +1 / \alpha_{1} * h * \exp \left(-\alpha_{1} *\left(u+w_{0}\right)\right) \\
& +\left(-2+\alpha_{1} * w_{1}+\alpha_{1} * u+\alpha_{1} * w_{0}\right) / \alpha_{1} * h
\end{aligned}
$$

Period 2:

$$
\begin{aligned}
I_{2}= & 1 / 2\left(2+2 \alpha_{2} u+2 \alpha_{2} w_{0}\right) / \alpha_{2} h \exp \left(-\alpha_{2}\left(u+w_{0}+w_{1}\right)\right)+\exp \left(-\alpha_{2}\left(u+w_{0}\right)\right) / \alpha_{2} h \\
& +\left(1 / 2\left(2+\alpha_{2}^{2} u^{2}+2 \alpha_{2}^{2} w_{1} u+2 \alpha_{2}^{2} u w_{0}+2 \alpha_{2} u+2 \alpha_{2} w_{1}+\alpha_{2}^{2} w_{0}^{2}\right.\right. \\
& \left.+2 \alpha_{2} w_{0}+2 \alpha_{2}^{2} w_{1} w_{0}\right) / \alpha_{2} h+1 / 2\left(2+\alpha_{2}^{2} u^{2}+2 \alpha_{2}^{2} w_{1} u+2 \alpha_{2}^{2} u w_{0}\right. \\
& \left.\left.+2 \alpha_{2} u+2 \alpha_{2} w_{1}+\alpha_{2}^{2} w_{0}^{2}+2 \alpha_{2} w_{0}+2 \alpha_{2}^{2} w_{1} w_{0}\right) / \alpha_{2} s\right) \exp \left(-\alpha_{2}\left(u+w_{0}+w_{1}\right.\right. \\
& +w w 2))+1 / 2\left(-6+2 \alpha_{2} u+2 \alpha_{2} w_{1}+2 \alpha_{2} w w 2+2 \alpha_{2} w_{0}\right) / \alpha_{2} h
\end{aligned}
$$

\section{Period 3:}




$$
\begin{aligned}
I_{3}= & 1 / 6\left(6 \alpha_{3}^{2} u w_{0}+6 \alpha_{3}^{2} w_{1} w_{0}+6 \alpha_{3}^{2} w_{1} u+6 \alpha_{3} w_{1}+3 \alpha_{3}^{2} w_{0}^{2}+6 \alpha_{3} u\right. \\
& \left.+6 \alpha_{3} w_{0}+3 \alpha_{3}^{2} u^{2}+6\right) / \alpha_{3} h \exp \left(-\left(u+w_{0}+w_{1}+w_{2}\right) \alpha_{3}\right) \\
& +1 / 6\left(6+6 \alpha_{3} u+6 \alpha_{3} w_{0}\right) / \alpha_{3} h \exp \left(-\left(u+w_{0}+w_{1}\right) \alpha_{3}\right) \\
& +\left(1 / 6\left(6 \alpha_{3} u+6 \alpha_{3} w_{0}+6+6 \alpha_{3} w_{2}+6 \alpha_{3} w_{1}+6 \alpha_{3}^{2} w_{2} w_{1}\right.\right. \\
& +3 \alpha_{3}^{2} w_{1}^{2}+3 \alpha_{3}^{2} u^{2}+3 \alpha_{3}^{2} w_{0}^{2}+6 \alpha_{3}^{3} w_{2} u w_{0}+3 \alpha_{3}^{3} w_{1}^{2} u+3 \alpha_{3}^{3} w_{0} u^{2} \\
& +3 \alpha_{3}^{3} w_{1} w_{0}^{2}+6 \alpha_{3}^{2} w_{2} u+3 \alpha_{3}^{3} w_{1} u^{2}+\alpha_{3}^{3} w_{0}^{3}+3 \alpha_{3}^{3} w_{0}^{2} u \\
& +3 \alpha_{3}^{3} w_{2} w_{0}^{2}+6 \alpha_{3}^{3} w_{1} u w_{0}+\alpha_{3}^{3} u^{3}+6 \alpha_{3}^{3} w_{2} w_{1} w_{0}+6 \alpha_{3}^{3} w_{2} w_{1} u \\
& +6 \alpha_{3}^{2} w_{2} w_{0}+3 \alpha_{3}^{3} w_{2} u^{2}+3 \alpha_{3}^{3} w_{1}^{2} w_{0}+6 \alpha_{3}^{2} w_{1} u \\
& \left.+6 \alpha_{3}^{2} w_{1} w_{0}+6 \alpha_{3}^{2} u w_{0}\right) / \alpha_{3} h+1 / 6\left(6 \alpha_{3} u+6 \alpha_{3} w_{0}+6\right. \\
& +6 \alpha_{3} w_{2}+6 \alpha_{3} w_{1}+6 \alpha_{3}^{2} w_{2} w_{1}+3 \alpha_{3}^{2} w_{1}^{2}+3 \alpha_{3}^{2} u^{2}+3 \alpha_{3}^{2} w_{0}^{2} \\
& +6 \alpha_{3}^{3} w_{2} u w_{0}+3 \alpha_{3}^{3} w_{1}^{2} u+3 \alpha_{3}^{3} w_{0} u^{2}+3 \alpha_{3}^{3} w_{1} w_{0}^{2} \\
& +6 \alpha_{3}^{2} w_{2} u+3 \alpha_{3}^{3} w_{1} u^{2}+\alpha_{3}^{3} w_{0}^{3}+3 \alpha_{3}^{3} w_{0}^{2} u+3 \alpha_{3}^{3} w_{2} w_{0}^{2} \\
& +6 \alpha_{3}^{3} w_{1} u w_{0}+\alpha_{3}^{3} u^{3}+6 \alpha_{3}^{3} w_{2} w_{1} w_{0}+6 \alpha_{3}^{3} w_{2} w_{1} u+6 \alpha_{3}^{2} w_{2} w_{0}+3 \alpha_{3}^{3} w_{2} u \\
& \left.\left.+3 \alpha_{3}^{3} w_{1}^{2} w_{0}+6 \alpha_{3}^{2} w_{1} u+6 \alpha_{3}^{2} w_{1} w_{0}+6 \alpha_{3}^{2} u w_{0}\right) / \alpha_{3} s\right) \exp \left(-\alpha_{3}\left(u+w_{0}\right.\right. \\
& \left.\left.+w_{1}+w_{2}+w_{3}\right)\right)+\exp \left(-\alpha_{3}\left(u+w_{0}\right)\right) / \alpha_{3} h+1 / 6\left(6 \alpha_{3} u+6 \alpha_{3} w_{0}\right. \\
& \left.+6 \alpha_{3} w_{3}-24+6 \alpha_{3} w_{2}+6 \alpha_{3} w_{1}\right) / \alpha_{3} h
\end{aligned}
$$

\section{Period 4:}

$I_{4}=\left(\left(\alpha_{4}^{2} u w_{0}+\alpha_{4}^{2} w_{2} u+\alpha_{4}^{2} w_{3} u+\alpha_{4}^{2} w_{3} w_{0}+1+\alpha_{4}^{2} w_{1} u+\alpha_{4}^{2} w_{1} w_{0}+\alpha_{4}^{2} w_{2} w_{0}+\right.\right.$ $\alpha_{4} w_{3}+\alpha_{4} w_{1}+\alpha_{4} w_{0}+\alpha_{4} u+\alpha_{4}^{3} w_{2} u w_{0}+\alpha_{4}^{3} w_{3} w_{1} w_{0}+\alpha_{4}^{3} w_{3} w_{1} u+\alpha_{4}^{3} w_{3} w_{2} w_{0}+$ $\alpha_{4}^{3} w_{3} u w_{0}+\alpha_{4}^{3} w_{1} u w_{0}+\alpha_{4}^{4} w_{3} w_{1} w_{0} u+\alpha_{4}^{4} w_{3} w_{2} u w_{0}+\alpha_{4} w_{2}+\alpha_{4}^{2} w_{3} w_{2}+\alpha_{4}^{3} w_{2} w_{1} w_{0}+$ $\alpha_{4}^{3} w_{2} w_{1} u+\alpha_{4}^{3} w_{3} w_{2} u+\alpha_{4}^{2} w_{2} w_{1}+\alpha_{4}^{2} w_{3} w_{1}+\alpha_{4}^{3} w_{3} w_{2} w_{1}+\alpha_{4}^{4} w_{2} w_{1} w_{0} u+\alpha_{4}^{4} w_{3} w_{2} w_{1} u+$ $\alpha_{4}^{4} w_{3} w_{2} w_{1} w_{0}+1 / 2\left(\alpha_{4}^{2} u+\alpha_{4}^{3} w_{1} u+\alpha_{4}^{3} w_{2} w_{0}^{2}+\alpha_{4}^{3} w_{0}^{2} u+\alpha_{4}^{3} w_{1}^{2} u+\alpha_{4}^{3} w_{2}^{2} w_{0}+\right.$ $\alpha_{4}^{3} w_{3} w_{0}^{2}+\alpha_{4}^{3} w_{1} w_{0}^{2}+\alpha_{4}^{3} w_{1}^{2} w_{0}+\alpha_{4}^{3} w_{2}^{2} w_{1}$

$+\alpha_{4}^{3} w_{2}^{2} u+\alpha_{4}^{3} w_{3} u+\alpha_{4}^{3} w_{2} u+\alpha_{4}^{3} w_{0} u+\alpha_{4}^{3} w_{2} w_{1}^{2}+\alpha_{4}^{4} w_{1} u w_{0}+\alpha_{4}^{4} w_{3} w_{0}^{2} u+$ $\alpha_{4}^{4} w_{3} w_{1} u+\alpha_{4}^{4} w_{2}^{2} w_{1} w_{0}+\alpha_{4}^{4} w_{2}^{2} w_{1} u+\alpha_{4}^{4} w_{3} w_{2} w_{0}^{2}+\alpha_{4}^{4} w_{3} w_{1} w_{0}^{2}+\alpha_{4}^{3} w_{3} w_{1}^{2}+\alpha_{4}^{2} w_{1}^{2}+$ $\alpha_{4}^{2} w_{2}^{2}+\alpha_{4}^{4} w_{2} w_{1}^{2} w_{0}+\alpha_{4}^{4} w_{2}^{2} w_{0} u+\alpha_{4}^{4} w_{2} w_{1} u+\alpha_{4}^{4} w_{2} w_{1} w_{0}^{2}+\alpha_{4}^{4} w_{3} w_{1}^{2} u+\alpha_{4}^{4} w_{1}^{2} u w_{0}+$ $\alpha_{4}^{4} w_{3} u w_{0}+\alpha_{4}^{4} w_{1} u w_{0}^{2}+\alpha_{4}^{4} w_{2} w_{1}^{2} u+\alpha_{4}^{4} w_{2} u w_{0}^{2}+\alpha_{4}^{4} w_{2} u w_{0}+\alpha_{4}^{4} w_{3} w_{1}^{2} w_{0}+\alpha_{4}^{4} w_{3} w_{2} u+$ $\left.\alpha_{4}^{2} w_{0}^{2}\right)+1 / 4\left(\alpha_{4}^{4} w_{1}^{2} u+\alpha_{4}^{4} w_{2}^{2} w_{0}^{2}+\alpha_{4}^{4} w_{2}^{2} u+\alpha_{4}^{4} u w_{0}^{2}+\alpha_{4}^{4} w_{1}^{2} w_{0}^{2}\right)$

$+1 / 6\left(\alpha_{4}^{4} u^{3} w_{0}+\alpha_{4}^{4} u w_{0}^{3}+\alpha_{4}^{4} w_{1} u^{3}+\alpha_{4}^{4} w_{3} u^{3}+\alpha_{4}^{4} w_{1}^{3} u+\alpha_{4}^{3} w_{0}^{3}+\alpha_{4}^{3} u^{3}+\alpha_{4}^{4} w_{1}^{3} w_{0}+\right.$ $\left.\left.\alpha_{4}^{4} w_{2} u^{3}+\alpha_{4}^{4} w_{2} w_{0}^{3}+\alpha_{4}^{4} w_{3} w_{0}^{3}+\alpha_{4}^{4} w_{1} w_{0}^{3}+\alpha_{4}^{3} w_{1}^{3}\right)+1 / 24\left(\alpha_{4}^{4} w_{0}^{4}+1 / 24 \alpha_{4}^{4} u^{4}\right)\right) / \alpha_{4} h+$ 
$1 / 24\left(\alpha_{4}^{4} w_{0}^{4}+\alpha_{4}^{4} u^{4}+24+12\left(\alpha_{4}^{2} u+\alpha_{4}^{3} w_{1} u+\alpha_{4}^{3} w_{2} w_{0}^{2}+\alpha_{4}^{3} w_{0}^{2} u+\alpha_{4}^{3} w_{1}^{2} u+\alpha_{4}^{3} w_{2}^{2} w_{0}+\right.\right.$ $\alpha_{4}^{3} w_{3} w_{0}^{2}+\alpha_{4}^{3} w_{1} w_{0}^{2}+\alpha_{4}^{3} w_{1}^{2} w_{0}+\alpha_{4}^{3} w_{2}^{2} u+\alpha_{4}^{3} w_{3} u+\alpha_{4}^{3} w_{2} u+\alpha_{4}^{3} w_{0} u+\alpha_{4}^{4} w_{2}^{2} w_{1} w_{0}+$ $\alpha_{4}^{4} w_{2}^{2} w_{1} u+\alpha_{4}^{3} w_{2}^{2} w_{1}+\alpha_{4}^{3} w_{2} w_{1}^{2}+\alpha_{4}^{4} w_{1} u w_{0}+\alpha_{4}^{4} w_{3} w_{0}^{2} u+\alpha_{4}^{4} w_{3} w_{1} u+\alpha_{4}^{4} w_{3} w_{2} w_{0}^{2}+$ $\alpha_{4}^{4} w_{3} w_{1} w_{0}^{2}+\alpha_{4}^{3} w_{3} w_{1}^{2}+\alpha_{4}^{2} w_{1}^{2}+\alpha_{4}^{2} w_{2}^{2}+\alpha_{4}^{4} w_{2} w_{1}^{2} w_{0}$

$+\alpha_{4}^{4} w_{2}^{2} w_{0} u+\alpha_{4}^{4} w_{2} w_{1} u+\alpha_{4}^{4} w_{2} w_{1} w_{0}^{2}+\alpha_{4}^{4} w_{3} w_{1}^{2} u+\alpha_{4}^{4} w_{1}^{2} u w_{0}+\alpha_{4}^{4} w_{3} u w_{0}+$ $\left.\alpha_{4}^{4} w_{1} u w_{0}^{2}+\alpha_{4}^{4} w_{2} w_{1}^{2} u+\alpha_{4}^{4} w_{2} u w_{0}^{2}+\alpha_{4}^{4} w_{2} u w_{0}+\alpha_{4}^{4} w_{3} w_{1}^{2} w_{0}+\alpha_{4}^{4} w_{3} w_{2} u+\alpha_{4}^{2} w_{0}^{2}\right)+$ $4\left(\alpha_{4}^{3} w_{0}^{3}+\alpha_{4}^{3} u^{3}+\alpha_{4}^{4} u w_{0}^{3}+\alpha_{4}^{4} w_{1} u^{3}+\alpha_{4}^{4} w_{3} u^{3}+\alpha_{4}^{4} w_{1}^{3} u+\alpha_{4}^{4} w_{1}^{3} w_{0}+\alpha_{4}^{4} w_{2} u^{3}+\right.$ $\left.\alpha_{4}^{4} w_{2} w_{0}^{3}+\alpha_{4}^{4} w_{3} w_{0}^{3}+\alpha_{4}^{4} u^{3} w_{0}+\alpha_{4}^{4} w_{1} w_{0}^{3}+\alpha_{4}^{3} w_{1}^{3}\right)+24\left(\alpha_{4}^{2} w_{1} u+\alpha_{4}^{2} w_{1} w_{0}+\alpha_{4}^{2} u w_{0}+\right.$ $\alpha_{4}^{2} w_{2} u+\alpha_{4}^{2} w_{3} u+\alpha_{4}^{2} w_{3} w_{0}+\alpha_{4}^{2} w_{2} w_{0}+\alpha_{4} w_{3}+\alpha_{4} w_{1}+\alpha_{4} w_{0}$

$+\alpha_{4} u+\alpha_{4}^{3} w_{2} u w_{0}+\alpha_{4}^{3} w_{3} w_{1} w_{0}+\alpha_{4}^{3} w_{3} w_{1} u+\alpha_{4}^{3} w_{3} w_{2} w_{0}+\alpha_{4}^{3} w_{3} u w_{0}+\alpha_{4}^{3} w_{1} u w_{0}+$ $\alpha_{4}^{3} w_{2} w_{1} w_{0}+\alpha_{4}^{3} w_{2} w_{1} u+\alpha_{4}^{3} w_{3} w_{2} u+\alpha_{4}^{2} w_{2} w_{1}+\alpha_{4}^{2} w_{3} w_{1}+\alpha_{4}^{3} w_{3} w_{2} w_{1}+\alpha_{4}^{4} w_{3} w_{2} u w_{0}+$ $\left.\alpha_{4} w_{2}+\alpha_{4}^{2} w_{3} w_{2}+\alpha_{4}^{4} w_{2} w_{1} w_{0} u+\alpha_{4}^{4} w_{3} w_{2} w_{1} u+\alpha_{4}^{4} w_{3} w_{2} w_{1} w_{0}+\alpha_{4}^{4} w_{3} w_{1} w_{0} u\right)+$ $\left.\left.6\left(\alpha_{4}^{4} w_{1}^{2} u+\alpha_{4}^{4} w_{2}^{2} w_{0}^{2}+\alpha_{4}^{4} w_{2}^{2} u+\alpha_{4}^{4} u w_{0}^{2}+\alpha_{4}^{4} w_{1}^{2} w_{0}^{2}\right)\right) / \alpha_{4} s\right) \exp \left(-\alpha_{4}\left(u+w_{0}+w_{1}+\right.\right.$ $\left.\left.w_{2}+w_{3}+w_{4}\right)\right)+1 / \alpha_{4} \exp \left(-\alpha_{4}\left(u+w_{0}\right)\right) h+1 / 24\left(24+24 \alpha_{4} u+24 \alpha_{4} w_{0}\right) / \alpha_{4} h \exp (-(u+$ $\left.\left.w_{0}+w_{1}\right) \alpha_{4}\right)+1 / 24\left(24\left(\alpha_{4} w_{1}+\alpha_{4}^{2} w_{1} u+\alpha_{4} w_{0}+\alpha_{4} u\right.\right.$

$\left.\left.+1+\alpha_{4}^{2} w_{1} w_{0}+\alpha_{4}^{2} u w_{0}\right)+12\left(\alpha_{4}^{2} u+\alpha_{4}^{2} w_{0}^{2}\right)\right) / \alpha_{4} h \exp \left(-\left(u+w_{0}+w_{1}+w_{2}\right) \alpha_{4}\right)+$ $1 / 24\left(12\left(\alpha_{4}^{2} u+\alpha_{4}^{3} w_{1} u+\alpha_{4}^{3} w_{2} w_{0}^{2}+\alpha_{4}^{3} w_{0}^{2} u \alpha_{4}^{3} w_{1}^{2} u+\alpha_{4}^{3} w_{1} w_{0}^{2}+\alpha_{4}^{3} w_{1}^{2} w_{0}+\alpha_{4}^{3} w_{2} u+\right.\right.$ $\left.\alpha_{4}^{3} w_{0} u+\alpha_{4}^{2} w_{1}^{2}+\alpha_{4}^{2} w_{0}^{2}\right)+4\left(\alpha_{4}^{3} w_{0}^{3}+\alpha_{4}^{3} u^{3}\right)+24\left(1+\alpha_{4}^{2} w_{1} u+\alpha_{4}^{2} w_{1} w_{0}+\alpha_{4}^{2} u w_{0}+\right.$ $\alpha_{4}^{2} w_{2} u+\alpha_{4}^{2} w_{2} w_{0}+\alpha_{4} w_{1}+\alpha_{4} w_{0}+\alpha_{4} u+\alpha_{4}^{3} w_{2} u w_{0}+\alpha_{4}^{3} w_{1} u w_{0}+\alpha_{4}^{3} w_{2} w_{1} w_{0}+$ $\left.\left.\alpha_{4}^{3} w_{2} w_{1} u+\alpha_{4}^{2} w_{2} w_{1}\right)+24 \alpha_{4} w_{2}\right) / \alpha_{4} h \exp \left(-\left(u+w_{0}+w_{1}+w_{2}+w_{3}\right) \alpha_{4}\right)+\left(\alpha_{4} w_{3}+\right.$ $\left.\alpha_{4} w_{1}+\alpha_{4} w_{0}+\alpha_{4} u+\alpha_{4} w_{2}+\alpha_{4} w_{4}-5\right) / \alpha_{4} h$ 


\section{Appendix 2: $\quad$ Simulation}

The inventory cost function is a complex expression. To verify if the expression was obtained in a correct form, we have ran a simulation.

For M1 and M2, we have picked up a retailer and solve the optimization problem.

And simulated the demand with the same behaviour (same mean and same standard deviation) for 10000 iterations and compared the solution obtained by optimization with the simulated one. The simualtion converges to the optimal solution, the slightly difference is due to the conversion of continuous to dicrete deliveries.

Model 1

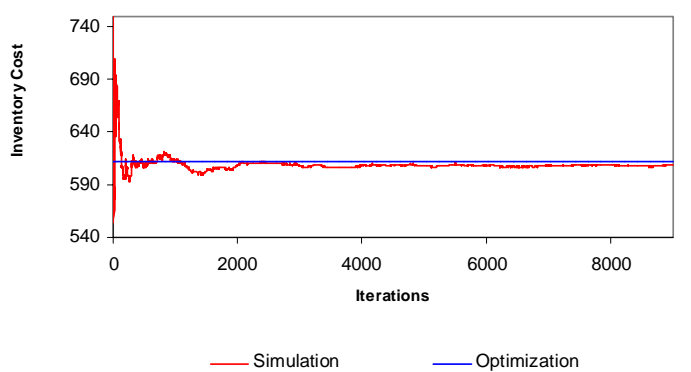

Model 2

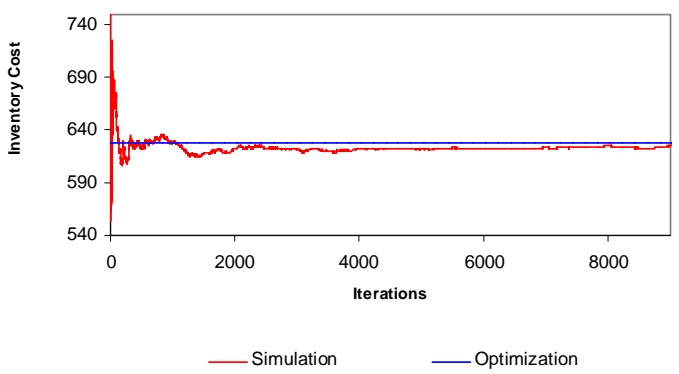

Figure 3: Simulation versus Optimization, for M1 and M2, for one customer. 